\title{
Irreversible Thermal Denaturation of Cytochrome $c$ Studied by Electrospray Mass Spectrometry
}

\author{
Jiangjiang Liu and Lars Konermann \\ Department of Chemistry, The University of Western Ontario, London, Ontario, Canada
}

This work uses electrospray ionization mass spectrometry (ESI-MS) in conjunction with hydrogen/deuterium exchange (HDX) and optical spectroscopy for characterizing the solutionphase properties of cytochrome $c$ (cyt $c$ ) after heat exposure. Previous work demonstrated that heating results in irreversible denaturation for a subpopulation of proteins in the sample. However, that study did not investigate the physical reasons underlying this interesting effect. Here we report that the formation of oxidative modifications at elevated temperature plays a key role for the observed behavior. Tryptic digestion followed by tandem mass spectrometry is used to identify individual oxidation sites. Trp59 and Met80 are among the modified amino acids. In native cyt $c$ both of these residues are buried deep within the protein structure, such that covalent modifications would be expected to be particularly disruptive. ESI-MS analysis after heat exposure results in a bimodal charge-state distribution. Oxidized protein appears predominantly in charge states around $11+$, whereas a considerably lower degree of oxidation is observed for the $7+$ and $8+$ peaks. This finding confirms that different oxidation levels are associated with different solution-phase conformations. HDX measurements for different charge states are complicated by peak distortions arising from oxygen adduction. Nonetheless, comparison with simulated peak shapes clearly shows that the HDX properties are different for high- and low-charge states, confirming that interconversion between unfolded and folded conformers is blocked in solution. In addition to oxidation, partial aggregation upon heat exposure likely contributes to the formation of irreversibly denatured protein. (J Am Soc Mass Spectrom 2009, 20, 819-828) ( 2009 American Society for Mass Spectrometry

S tudies on the structure and dynamics of proteins continue to be a focal area of biochemical research. A number of well-established tools are available for experiments of this kind, including X-ray crystallography, nuclear magnetic resonance, calorimetry, and various spectroscopic techniques. Electrospray ionization mass spectrometry (ESI-MS) has become another widely used approach for exploring the properties of proteins in solution, providing information complementary to that obtained by traditional methods [1]. The ESI process generates intact gas-phase ions from proteins in solution. In the commonly used positive-ion mode these $[\mathrm{M}+n \mathrm{H}]^{n+}$ species are multiply charged because of proton attachment.

ESI of natively folded proteins results in protonation states $n$ similar to those predicted for water droplets of the same size that are charged to the Rayleigh limit. This finding supports the idea that ionization follows the charged-residue model [2-6], although alternative scenarios have been proposed as well [7-9]. Much higher protonation states and wider charge-state distri-

Address reprint requests to Dr. Lars Konermann, The University of Western Ontario, Department of Chemistry, London, ON, Canada N6A 5B7. E-mail: konerman@uwo.ca butions are generally seen for proteins that are unfolded in solution. Based on this empirical relationship, ESI-MS is now routinely being used for monitoring structural transitions in response to changes in $\mathrm{pH}$, temperature, organic co-solvents, or covalent modifications [1, 10-16]. The physical basis for the striking dependence of ESI charge states on the solution-phase protein conformation continues to be a matter of debate. Various explanations have been put forward, including differences in the steric accessibility of protonation sites and changes of the corresponding $\mathrm{p} K_{\mathrm{a}}$ values [10,17]. It has also been argued that more extended conformations reduce the extent of coulombic repulsion in multiply protonated ions [18]. In addition, the enhanced structural flexibility of unfolded proteins can favor intramolecular solvation of charged sites $[19,20]$. Other proposals invoke differences in gas-phase basicity between protein and solvent [21]. Mechanisms involving conformation-dependent charge neutralization [22] or combinations of electrostatic and steric shielding [23] have been put forward, as well as proposals that ESI charge states reflect the protein surface area [24], and that they specifically monitor changes in tertiary (not secondary) structure [11]. 
Using ESI charge-state distributions for protein structural studies offers a number of unique advantages. Differentiating coexisting conformers can be challenging when using traditional spectroscopic techniques, whereas multimodal ESI charge-state distributions permit the direct visualization of individual species $[25,26]$. The gentle nature of the ESI process allows the transfer of intact protein-ligand and proteinprotein complexes into the gas phase [6, 27-29]; thus, insights into conformations and interactions are obtained in a single experiment [17, 30-36]. A further dimension can be added to these studies by incorporating ion mobility spectrometry $[37,38]$.

A very interesting approach is to monitor the chargestate distribution of a protein, while simultaneously measuring its hydrogen/deuterium exchange (HDX) behavior [39-42]. The two probes complement each other, given that ESI charge states report on the "compactness" of a protein in solution [20], whereas HDX is governed largely by the hydrogen-bonding behavior along the amide backbone. HDX at sites that are involved in stable hydrogen bonds or that are sterically shielded is mediated by conformational fluctuations. Thus, proteins that are disordered and highly mobile undergo much faster isotope exchange than tightly folded conformers [43].

Because the individual components of bi- or multimodal ESI charge-state distributions represent distinct solution-phase conformers, it is not surprising that different charge states in a single spectrum can be associated with different HDX properties [41, 44-48]. However, not all cases show this behavior. Several studies have found that charge states representing very different coexisting protein structures can exhibit isotope exchange kinetics that are indistinguishable [4951]. This conundrum is resolved when it is considered that coexisting conformers may interconvert in solution [52]. All species will exhibit the same HDX kinetics if interconversion is rapid on the HDX timescale. This relationship was first proposed in a seminal study by Wagner and Anderegg [53]. In that work it was reported that cytochrome $c$ (cyt $c$ ) under mildly acidic conditions exhibits a bimodal charge-state distribution, representing the presence of unfolded proteins in addition to the native conformers. The two forms showed very similar isotope exchange kinetics. The experiment was then repeated on samples that had been partially denatured in an irreversible manner by heat exposure. When the heated samples were transferred back to a native solvent environment at room temperature a bimodal charge-state distribution was found to persist, corresponding to a mix of permanently unfolded protein and natively folded polypeptide chains. With interconversion being blocked, it was reported that highcharge states showed more extensive HDX than did low-charge states. Those widely cited results [53] were instrumental in establishing ESI charge states as a well-accepted probe for protein conformational studies.
Most unfolded proteins will spontaneously refold to the native state once placed in a non-denaturing solvent environment, i.e., ambient temperature, neutral $\mathrm{pH}$, and in the absence of chemical denaturants [54]. A key question that was not explicitly addressed in Wagner and Anderegg's study [53] and in related previous work [55] is why heat exposure of cyt $c$ results in the formation of conformers that are irreversibly unfolded. The reasons underlying the apparently different HDX characteristics for high- and low-charge states thus remain unclear. Irreversible thermal denaturation has also been observed using other techniques for a number of different proteins. It was proposed that this phenomenon may be caused by a combination of factors, including partial aggregation and various covalent modifications [56-61]. The current study revisits the experiments of Wagner and Anderegg [53], with the aim of providing additional insights into the ESI-MS and HDX characteristics of cyt $c$ after heat exposure. It is found that the formation of irreversibly denatured protein is linked to oxidative damage. Partial aggregation may play a role as well. Covalent oxygen adduction interferes with the analysis of HDX experiments, necessitating a re-examination of the question, irrespective of whether high- and low-charge states of heat-exposed cyt $c$ exhibit distinct isotope exchange properties.

\section{Experimental}

\section{Materials}

Bovine heart cyt $c(12,230 \mathrm{Da}$ [62]) was purchased from Sigma (St. Louis, MO, USA). Ammonium acetate and formic acid were from Fluka (St. Louis, MO, USA). Ammonia was obtained from Caledon Laboratory Chemicals (Caledon, $\mathrm{ON}$, Canada) and $\mathrm{D}_{2} \mathrm{O}$ was from Cambridge Isotope Laboratories (Andover, MA, USA). Thermally denatured protein was generated using procedures very similar to those of Wagner and Anderegg [53]. Aliquots $(25 \mathrm{~mL})$ containing $1 \mu \mathrm{M}$ cyt $c$ were heated in double-distilled water ( $\mathrm{pH}$ 6.5) for 1 or $2 \mathrm{~h}$ at $90{ }^{\circ} \mathrm{C}$ in a water bath. Higher protein concentrations resulted in unacceptable levels of aggregation. After heat exposure the samples were flash frozen in liquid nitrogen, lyophilized, and subsequently re-dissolved to $500 \mu \mathrm{L}$ in $5 \mathrm{mM}$ aqueous ammonium acetate. The $\mathrm{pH}$ was adjusted to 7 by addition of ammonia. UV-Vis measurements $\left(\varepsilon_{\text {Soret }}=1.06 \times 10^{5} \mathrm{M}^{-1} \mathrm{~cm}^{-1}\right.$ [63] $)$ indicate the loss of roughly $30 \%$ protein during the procedure, yielding a final stock solution of about 35 $\mu \mathrm{M}$. Native cyt $c$ samples were prepared in $5 \mathrm{mM}$ ammonium acetate at $\mathrm{pH} 7$ and acid-denatured protein was generated by addition of formic acid to $\mathrm{pH} 2$ (both without heating). Protein digestion was performed by trypsin spin columns (Sigma). The sequence of bovine cyt $c$ (pdb code $2 b 4 z$ [62]) along with its tryptic cleavage sites $(\downarrow)$ is indicated below:

${ }^{1} \mathrm{GDVEK} \downarrow \mathrm{GK} \downarrow \mathrm{K} \downarrow$ IF VQK $\downarrow$ CAQCHTV EK $\downarrow$ GGK $\downarrow$ HK $\downarrow$ TGP NLHGLFGR $\downarrow$ K $\downarrow T^{41}$ GQAPGFSYTD ANK $\downarrow$ 
NK $\downarrow$ GITWG EETLMEYLEN PK $\downarrow$ K $\downarrow$ YIPGTK $\downarrow$ M

${ }^{81}$ IFAGIK $\downarrow K \downarrow K \downarrow$ GE $R \downarrow$ EDLIAYLK $\downarrow$ K $\downarrow$ ATNE.

\section{Mass Spectrometry, Liquid Chromatography (LC)/ MS, and Hydrogen/Deuterium Exchange}

All mass spectra were acquired on a Q-ToF Ultima API mass spectrometer (Waters Corp., Milford, MA, USA), equipped with a Z-spray ESI source that was operated in positive-ion mode. The capillary and cone voltages were $3 \mathrm{kV}$ and $60 \mathrm{~V}$, respectively, and the rangefinder (RF) lens 1 was set to $20 \mathrm{~V}$, unless indicated otherwise. Cone and desolvation gas flow rates were 50 and $500 \mathrm{~L}$ $\mathrm{h}^{-1}$, respectively. The desolvation temperature was set to $120^{\circ} \mathrm{C}$ and that of the source to $80^{\circ} \mathrm{C}$. For characterizing protein charge-state distributions, cyt $c$ was infused directly into the ion source at a flow rate of $5 \mu \mathrm{L}$ $\min ^{-1}$ using a syringe pump (Harvard Apparatus, Boston, MA, USA). The protein concentrations used were $5 \mu \mathrm{M}$ for native and acid-denatured cyt $c$, whereas the low signal intensities after heat exposure necessitated a higher protein concentration of $35 \mu \mathrm{M}$. LC/MS experiments were conducted using a Waters $1525 \mu$ HPLC pump with a C18 (Symmetry 300) $2.1 \times 100-\mathrm{mm}$ reversed-phase column at a flow rate of $100 \mu \mathrm{L} \mathrm{min}{ }^{-1}$ and a water/acetonitrile gradient in the presence of $0.1 \%$ formic acid. The injection loop volume was $25 \mu \mathrm{L}$ at a protein concentration of 25 to $35 \mu \mathrm{M}$. Identities of tryptic peptides were confirmed by tandem mass spectrometry (MS/MS). These measurements were carried out in data-dependent acquisition mode, where the instrument automatically switches to MS/MS once a peptide of interest elutes from the column. HDX experiments were performed by mixing protein solution with $\mathrm{D}_{2} \mathrm{O}$ in a 1:2 ratio. The mixture was transferred into a syringe and directly infused into the ion source of the mass spectrometer. The $x$-axis was converted from $\mathrm{m} / \mathrm{z}$ to mass $M$ for individual ionic signals according to $M=$ $(m / z \times n)-(0.667 \times 2.014 \times n)-(0.333 \times 1.008 \times n)$, where $n$ is the charge state of the corresponding ion. Bovine cyt $c$ contains a total of 192 exchangeable hydrogens [53]. Hydrogen back exchange in the gas phase is negligible under the conditions used here [64].

\section{Optical Spectroscopy}

UV-Vis absorption spectra were recorded on a Varian Cary 100 spectrophotometer (Palo Alto, CA, USA). To facilitate the comparison of spectra obtained under different conditions the data were normalized to unity at the Soret maximum. Fluorescence emission spectra were acquired on a Fluorolog-3 instrument (Horiba Jobin Yvon, Edison, NJ, USA), using an excitation wavelength of $280 \mathrm{~nm}$ and protein concentrations that were normalized to $5 \mu \mathrm{M}$. Protein-free solutions were used as blanks in all absorption and fluorescence experiments. Circular dichroism (CD) spectra were recorded on a Jasco J-810 spectropolarimeter (Easton, MD, USA) with a 1-mm path-length cuvette. The protein concentration used for $\mathrm{CD}$ experiments was $35 \mu \mathrm{M}$ and the measured spectra were converted to mean residue ellipticity $[53,65]$. $\mathrm{HCl}$ was used instead of formic acid for CD measurements on acid-denatured cyt $c$ to avoid complications related to excessive light absorption in the far UV.

\section{Results and Discussion}

ESI-MS, optical spectroscopy, and HDX measurements were used to examine the effects of heat exposure on the structure of cyt $c$. Samples that had undergone thermal denaturation and subsequent lyophilization were compared to native cyt $c$ and to samples at $\mathrm{pH} 2(1.2 \%$ formic acid), where the protein is extensively unfolded [11]. Protein that had undergone heat exposure was studied in the same solvent environment as native cyt $c$ (i.e., $\mathrm{pH} \mathrm{7)}$ in $5 \mathrm{mM}$ ammonium acetate at room temperature $\left(22 \pm 1^{\circ} \mathrm{C}\right)$.

\section{ESI Charge-State Distributions}

The ESI mass spectrum of native cyt $c$ shows a narrow charge-state distribution that encompasses only the 7+ and $8+$ peaks, representing a tightly folded solutionphase conformation (Figure 1a). A much wider distribution centered around 15+ and extending all the way to $20+$ is observed for the acid-denatured protein at $\mathrm{pH}$ 2 (Figure 1d). These observations are in line with previous reports $[11,25,53]$. Protein that had been heat exposed for $1 \mathrm{~h}$ retains its most intense charge state at $8+$, but the relative intensity of the $7+$ peak is diminished. In addition, higher charge states extending up to at least $14+$ are observed (Figure 1b). The relative intensity of highly charged ions is further elevated for a heating period of $2 \mathrm{~h}$ (Figure 1c). The appearance of these spectra was found to be stable for days. Extending the heating period to more than $2 \mathrm{~h}$ resulted in data with increasingly poor signal-to-noise ratio (not shown). The results depicted in Figure $1 b$ and $c$ are consistent with earlier observations $[53,55]$ that heat exposure of cyt $c$ generates a subpopulation of proteins that are irreversibly unfolded. In the spectra of Figure $1 c$ and $d$, these non-native proteins give rise to charge states centered around $11+$.

Compared to the data in Figure $1 \mathrm{~b}$ and $\mathrm{c}$, somewhat higher charge states with considerably higher relative intensities were seen in Wagner and Anderegg [53] for heat-denatured conformers, an effect that is tentatively attributed to differences in instrumentation. Quadrupole instruments of the type used in Wagner and Anderegg [53] may discriminate against ions with high $\mathrm{m} / \mathrm{z}$ [66], resulting in skewed spectra when compared to the TOF-MS data reported here. Other instrument characteristics such as the ion source design could play a role as well. 


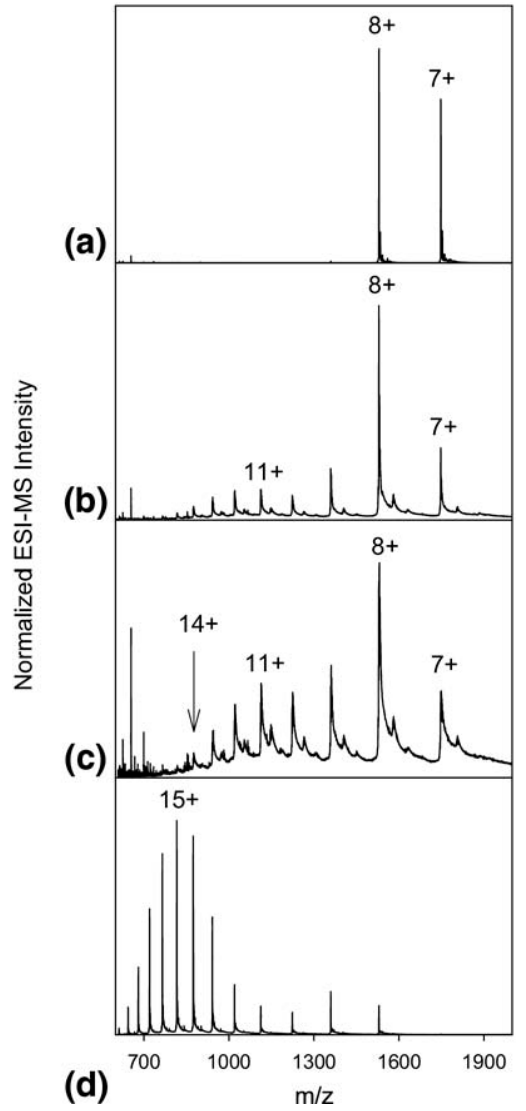

Figure 1. ESI mass spectra of bovine cyt $c$ recorded at room temperature under different conditions. (a) Native protein at $\mathrm{pH}$ 7; (b) after heat exposure for $1 \mathrm{~h}(\mathrm{pH} 7)$; (c) after heat exposure for $2 \mathrm{~h}$ (pH 7); (d) Acid denatured cyt $c$ at $\mathrm{pH} 2$ without prior heat exposure.

\section{Optical Spectroscopy}

Cyt $c$ represents a convenient model system because it offers several optical probes for monitoring the occurrence of structural changes. The UV-Vis absorption spectrum reports on the heme environment [11, 63]. Coordination of the heme iron with its native His18 and Met80 ligands leads to a Soret absorption maximum at $409 \mathrm{~nm}$. Displacement of these ligands upon acid unfolding induces a blue shift to $394 \mathrm{~nm}$. A much smaller shift to $407 \mathrm{~nm}$ is seen after $2 \mathrm{~h}$ of heat exposure (Figure 2a). A prominent feature in the spectrum of the heated protein is a sloped baseline that strongly increases for shorter wavelengths. This effect can be attributed to Rayleigh scattering caused by small particles that are suspended in solution, representing a hallmark of partial protein aggregation [61]. Unfortunately, quantifying the extent of aggregation is not straightforward because scattering is affected by both the particle size and the number density.

The CD spectrum of native cyt $c$ shows minima at 208 and $222 \mathrm{~nm}$, features that attest to a largely helical secondary structure (Figure $2 \mathrm{~b}$ ). In contrast, the aciddenatured protein has its main minimum at $202 \mathrm{~nm}$, which is consistent with the prevalence of random coil-like elements [67]. Also heat exposure induces some secondary structure perturbations, as evidenced by the differences between the solid and the dashed spectra in Figure $2 \mathrm{~b}$. The change in ellipticity at $222 \mathrm{~nm}$ between these two datasets suggests a $20 \%$ loss in helicity after heating, a finding that is consistent with the results of Wagner and Anderegg [53].

The single Trp residue (Trp59) in native cyt $c$ is virtually non-fluorescent as the result of efficient Förster resonance energy transfer to the heme, from where the excitation energy is dissipated as heat [11, 68]. Unfolding disrupts this quenching mechanism by increasing the heme-Trp distance. As a result, exposure of the protein to $\mathrm{pH} 2$ leads to a dramatic increase in fluorescence intensity. A fluorescence increase is also seen for cyt $c$ after heating, but the signal intensity in this case is only about $20 \%$ of that seen for the acidunfolded protein (Figure 2c).

The data presented so far confirm that heat exposure of cyt $c$ causes irreversible unfolding for a subpopulation of proteins in the sample. A shift to higher ESI charge states indicates a more expanded conformation. Changes in the UV-Vis and fluorescence spectra point
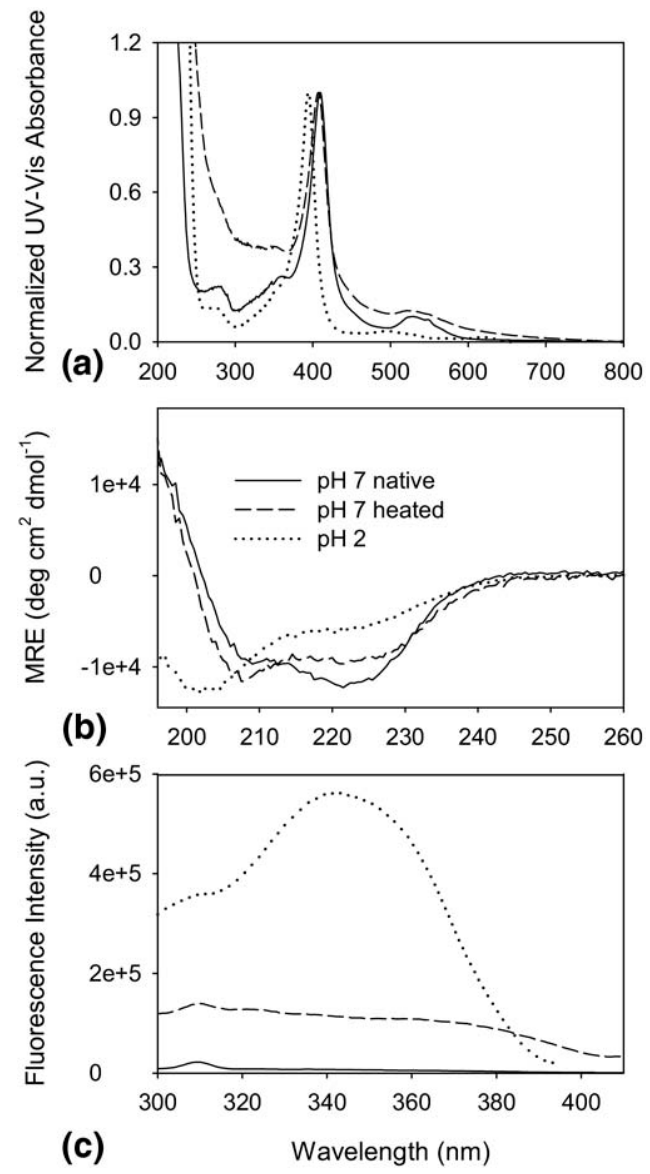

Figure 2. Optical spectra of native cyt $c$ at pH 7 (solid lines), after $2 \mathrm{~h}$ of heating at $\mathrm{pH} 7$ (dashed lines), and acid-denatured cyt $c$ at $\mathrm{pH} 2$ (dotted lines). (a) UV-Vis spectra, normalized to unity at the Soret maximum; (b) CD spectra, expressed as mean residue ellipticity (MRE); (c) fluorescence emission spectra. 
to tertiary structure alterations and CD spectroscopy shows a reduced $\alpha$ helicity. However, the overall magnitude of these heat-induced structural changes is moderate compared with that of the more dramatic effects seen upon acid-induced unfolding. Heat-exposed cyt $c$ aggregates to a certain extent, which is consistent with the behavior seen for other proteins [61]. Although non-native aggregates can sometimes be observed directly by ESI-MS $[20,34]$ this is not the case under the conditions of this work, even when extending the mass range for the measurements in Figure $1 \mathrm{~b}$ and c (data not shown).

\section{Oxidative Modifications}

A striking feature in the ESI mass spectra of thermally treated cyt $c$ (Figure $1 b$ and $c$ ) is the occurrence of extensive peak broadening and tailing, resulting in data with relatively poor signal-to-noise ratio. Close inspection of individual peaks reveals that the heated protein has undergone oxidative modifications. All charge states show a signal corresponding to the mass of unmodified cyt $c$, but there are additional satellite peaks shifted by $+16 \mathrm{Da}$ and multiples thereof (Figure 3a and b). Covalent modifications of this type may be induced by exposure to $\mathrm{H}_{2} \mathrm{O}_{2}$ or $\cdot \mathrm{OH}[69,70]$, but it has been demonstrated that oxidation can also occur during heat
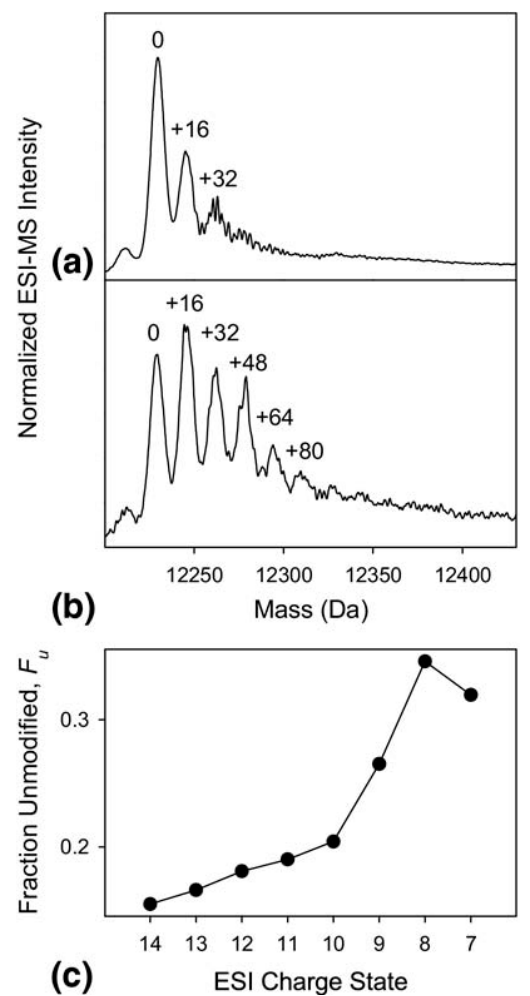

Figure 3. ESI-MS data for cyt $c$ after $1 \mathrm{~h}$ of heating. (a) $8+$ charge state plotted on a mass scale. (b) 13+ charge state plotted on a mass scale. Notation: " 0 " represents the mass of the unmodified protein; "+16," "+32," etc. represent adduction of one, two, etc. oxygens. (c) Oxidation level of individual charge states, expressed as fraction of unmodified protein, $F_{u}$. exposure in the absence of such reactive compounds [61]. The mechanisms of protein oxidation can be quite complex, but many reaction pathways result in the incorporation of oxygen atoms into amino acid side chains, thereby accounting for the observed $+16 \mathrm{Da}$ adducts [69]. Previous ESI-MS studies on thermally denatured cyt $c[53,55]$ did not address the possible occurrence of protein oxidation and close-ups of individual peaks were not shown. However, the spectra for heated samples in Wagner and Anderegg [53] and Mirza et al. [55] had a "noisy" appearance, reminiscent of the data in Figure $1 b$ and $c$, making it likely that oxidation also took place under the conditions of those previous investigations.

Thermally induced oxidation is less pronounced for low-charge states than that for highly charged protein ions. After $1 \mathrm{~h}$ of heating, for example, the base peak for cyt $c 7+$ and $8+$ corresponds to the mass of the unmodified protein. This is followed by smaller $+16 \mathrm{Da}$ and $+32 \mathrm{Da}$ adducts (Figure 3a). Residual unmodified cyt $c$ is also seen for higher charge states, but the most intense peak in these cases is for singly oxidized protein, followed by a progression up to at least 5-fold oxidation (Figure $3 \mathrm{~b}$ ). It is common to report protein oxidation levels as "fraction unmodified," $F_{u}$, defined as $A_{u} / A_{\text {tot }}$ [69]. In this expression $A_{u}$ is the peak area corresponding to the unmodified protein and $A_{t o t}$ is the area of the entire mass distribution including unmodified and oxidized species. Peak integration for determining $A_{\text {tot }}$ in the current work was performed over the mass range from zero to five oxidations. Analysis of ESI-MS data after $1 \mathrm{~h}$ of heating (Figure $1 \mathrm{~b}$ ) results in $F_{u}$ values around 0.35 for charge states $7+$ and $8+. F_{u}$ gradually decreases for higher charge states, down to a value of 0.16 for $14+$ (Figure 3c). Depending on their location within the protein, oxidative modifications can severely disrupt the native fold [71-73]. This is consistent with the observation that cyt $c$ with elevated oxidation levels is preferentially seen in high-charge states (Figure 3b). These data imply that oxidative modifications are a major contributing factor to the formation of irreversibly denatured cyt $c$ after heat exposure.

Tryptic peptide mapping was used for locating major modification sites within the heated protein. Some oxidation was found to occur within the region covered by peptide T12 (residues 56-72). Marker fragments $b_{4}+$ $16\left(\mathrm{~m} / \mathrm{z}\right.$ 474.2) and $\mathrm{y}_{8}{ }^{\prime \prime}+16(\mathrm{~m} / \mathrm{z}$ 1039.5) reveal oxygen incorporation at both Met65 and Trp59. Also T15 (residues 80-86) carries a +16 Da modification. Observation of $\mathrm{b}_{2}+16(\mathrm{~m} / \mathrm{z} 261.1)$, along with unmodified $\mathrm{y}_{6}{ }^{\prime \prime}(\mathrm{m} / \mathrm{z}$ 648.4) pinpoints Met80 as the site of oxidation in this segment (data not shown). These results for T12 and T15 are consistent with previous work that found Met and Trp to be among the amino acids with the highest intrinsic reactivities [69]. Oxidation was also observed for T4-5 (residues 9-22). Unfortunately, the interpretation of MS/MS spectra (not shown) for this peptide is complicated by the presence of heme, which is co- 


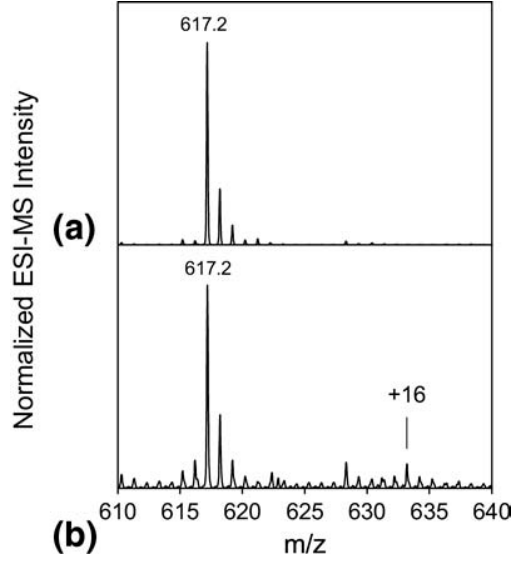

Figure 4. Partial ESI mass spectra recorded under "harsh" declustering conditions (cone voltage $150 \mathrm{~V}$, RF lens 1 voltage $170 \mathrm{~V}$ ), where [heme $+\mathrm{H}]^{+}$is released as collision induced dissociation product [74]. (a) Native protein; (b) after heat exposure for $1 \mathrm{~h}$.

valently attached to Cys14 and Cys17 via thioiether bonds. Fortunately, some insights can be obtained by collisional activation of the intact protein during ESI, leading to the formation of protonated heme as a major fragmentation product [74]. Collision-induced dissociation of non-heated cyt $c$ produces a clean heme signal at $\mathrm{m} / \mathrm{z} 617.2$ that is accompanied only by its isotope peaks (Figure 4a). Analysis of heat-exposed protein results in additional ions including (heme $+16 \mathrm{Da}$ ) and a host of other unidentified species (Figure 4b). This result strongly suggests that heme itself undergoes covalent modifications during heat exposure, an interpretation that is supported by studies on myoglobin under oxidizing conditions [75]. In addition to the modifications uncovered here, other sites of oxidation likely exist. Based on the known reactivity of sulfur-containing residues [69], the thioether groups of Cys14 and Cys17 in T4-5 represent possible candidates.

\section{Hydrogen/Deuterium Exchange}

Unheated cyt $c$ in $0.33 \%$ formic acid shows a bimodal charge-state distribution (not shown), representing the presence of co-populated unfolded and native proteins. As reported previously [53], exposing the protein under these conditions to $\mathrm{D}_{2} \mathrm{O}$ results in HDX kinetics that are very similar for all charge states. As an example, Figure 5 a shows mass distributions for the $7+$ and $13+$ peaks after $7 \mathrm{~min}$ of exchange. The peak maxima are shifted by 97 and $98 \mathrm{Da}$, respectively, relative to unlabeled cyt c. This corresponds to a relative HDX level around $97 /(0.66 \times 192)=77 \%$. As discussed earlier, the observation of virtually the same exchange kinetics for all charge states can be attributed to the rapid interconversion of native and non-native conformers under the conditions of partial acid unfolding [49-51,53].

A key finding in the study of Wagner and Anderegg [53] was the observation of different HDX mass shifts for high- and low-charge states of heated cyt $c$. Also in the current work the heat-exposed protein shows mass distributions for all $10+$ to $15+$ peaks that are very different from those of the $7+$ and $8+$ charge states (exemplified for $7+$ and $13+$ in Figure $5 \mathrm{~b}$ for $t \approx 17.5$ min). The accurate determination of HDX levels is

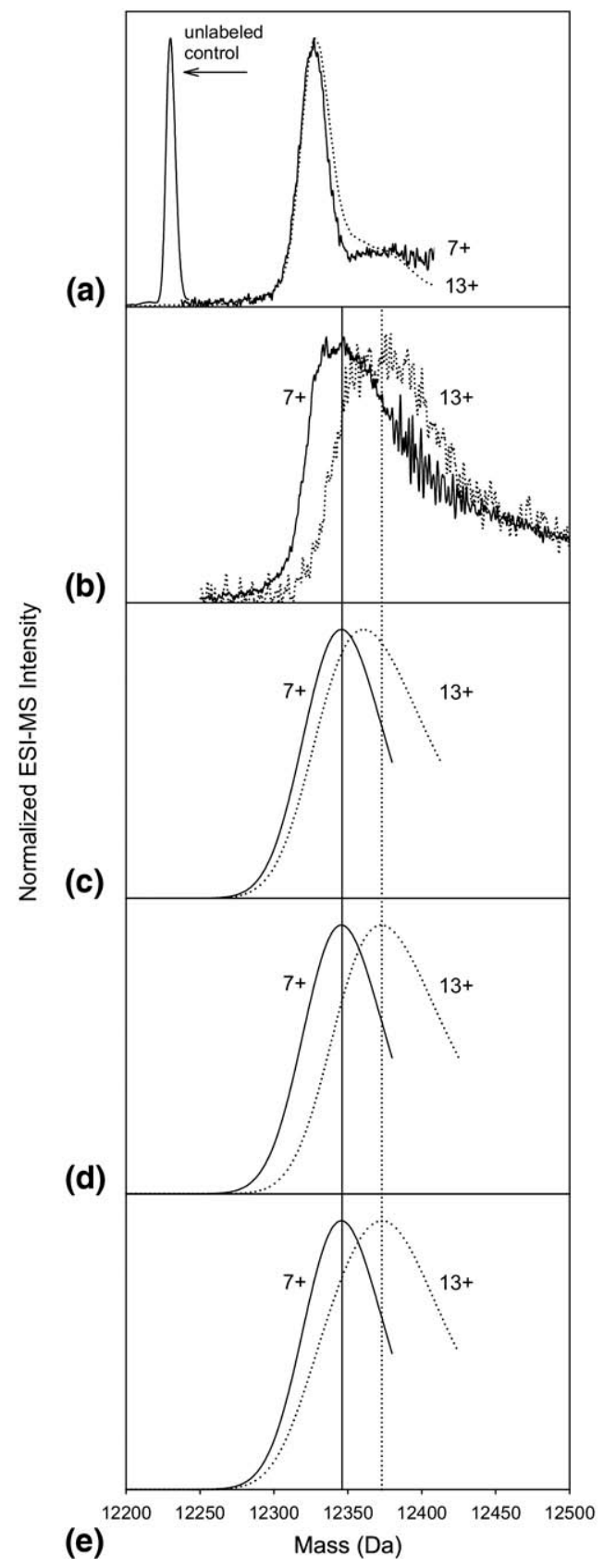

Figure 5. ESI mass distributions for two representative cyt $c$ charge states obtained after incubation in $66 \% \mathrm{D}_{2} \mathrm{O}$. Solid lines: $7+$, dotted lines: $13+$. (a) Partially denatured (unheated) cyt $c$ with $0.33 \%$ formic acid. Integration was performed for an HDX time window of $7 \pm 1 \mathrm{~min}$. (b) Cyt $c$ after $1 \mathrm{~h}$ of heating in neutral solution. Integration was performed for an HDX time window of $17.5 \pm 2.5 \mathrm{~min}$. Also shown in (a) is the mass distribution of the unlabeled protein. Panels (c), (d), and (e) represent different peak shape simulations, aimed at reproducing the experimental $7+$ and $13+$ data in (b). Details are provided in the text. Vertical lines indicate approximate centroids for the distributions in (b). 
complicated by peak broadening and by the limited signal-to-noise ratio of the data. From the approximate centroids (vertical lines in Figure 5b) it is estimated that high-charge states show a mass shift that is 25-30 Da larger than that for low-charge states. At this juncture it is tempting to directly follow the arguments of Wagner and Anderegg [53] and conclude that this difference is attributed to vastly different HDX characteristics, caused by non-interconverting folded and unfolded polypeptide chains in heat-exposed samples. However, this interpretation may be overly simplistic because the observed mass shifts result from a combination of HDX and protein oxidation. In other words, it is not immediately clear whether highly charged ions really show elevated HDX levels. Alternatively, the differences in Figure $5 \mathrm{~b}$ might be caused solely by the more extensive oxidation of highly charged ions (Figure $3 a$ and b), which will shift the mass envelope to the right even if all peaks showed the same HDX behavior. To examine whether such a "trivial" explanation can account for the effects seen in Figure $5 b$ we resort to peak shape simulations.

HDX induces centroid mass shifts in addition to peak broadening [76]. The mass distribution of a broadened peak $p$ after HDX may be approximated as a convolution of the protein's shifted mass profile $f$ with a Gaussian distribution function $D$ [64]. This can be expressed as

$$
p(M)=\left[f^{*} D\right](M)
$$

where

$$
\left[f^{*} D\right](M)=\int_{-\infty}^{\infty} f(\tau) D(M-\tau) d \tau
$$

A FORTRAN program was written for the numerical evaluation of eq 2. The procedure is illustrated in Figure 6 , where the mass profile $f$ is modeled as the sum of five Gaussians that are spaced by $16 \mathrm{Da}$. Width and intensity progression were chosen to mimic the $7+$ and $8+$ charge states of heated cyt $c$ (Figure 3a). The entire profile $f$ was then shifted by $106 \mathrm{Da}$ to account for a mass increase ascribed to HDX. A Gaussian band with a full width at half-maximum (FWHM) value of $50 \mathrm{Da}$ serves as the distribution function $D$. Convolution of $f$ and $D$ according to eq 2 results in an unstructured peak $p$. The presence of oxygen adducts $(+16,+32$, etc.) in $f$ causes $p$ to be slightly asymmetric, with a maximum that is shifted by an additional $10 \mathrm{Da}$ relative to the non-oxidized protein (indicated by the arrows in Figure 6). We will now examine a few hypothetical HDX scenarios based on the known oxidation patterns of high- and low-charge states in heated cyt $c$. The simulation procedure outlined in Figure 6 does not account for non-specific solvent adducts that are frequently observed on the high-mass side of ESI-MS peaks. Comparisons with experimental data will therefore focus on the low-mass portion of the simulated curves, not far past the peak maxima. To emphasize this point the

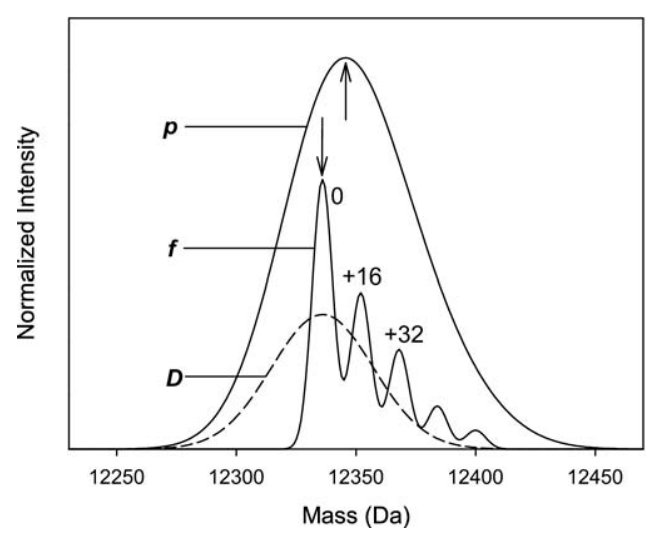

Figure 6. Illustration of how the peak shape $p$ is generated as convolution product of a mass profile $f$ and a Gaussian distribution function $D$. Labels $0,+16$, and +32 represent the degree of oxygen adduction as in Figure 3a. Arrows highlight the positions of peak maxima. Intensity scaling of the three curves relative to each other is arbitrary. Additional information is provided in the text.

simulated profiles will be displayed as truncated on the high-mass side.

Scenario a. We first consider a hypothetical scenario where all oxidation levels in high- and low-charge states show the same HDX behavior, corresponding to the "trivial" case outlined earlier. The parameters used for Figure 6 (HDX mass shift $=106 \mathrm{Da}$, FWHM of $D=$ $50 \mathrm{Da}$ ) provide a low-mass portion and peak maximum that are in reasonable agreement with the experimental $7+$ data for the heated protein (solid lines in Figure 5b and c). For reasons of simplicity, this parameter set will be retained for modeling the low-charge-state behavior in all cases considered here. Using a profile $f$ that mimics the elevated oxidation level of the high-charge states (Figure $3 b$ ) under otherwise identical simulation conditions results in a peak maximum that is shifted further to the right (dotted profile in Figure 5c). However, comparison with the experimental $13+$ data in Figure $5 \mathrm{~b}$ reveals that this simulated mass shift falls short of that seen for the measured $13+$ signal. It is concluded that the "trivial" case considered here cannot account for the experimentally observed difference in mass shift after HDX.

Scenario $b$. The previous scenario is now modified by assuming that the entire peak envelope of the $13+$ charge state undergoes an additional shift attributed to more extensive HDX. Acceptable agreement with the experimental data is obtained if this additional shift is chosen to be $12 \mathrm{Da}$ (dotted lines in Figure $5 \mathrm{~b}$ and $\mathrm{d}$ ). Under these conditions the measured centroids are well reproduced for both $7+$ and $13+$, as well as the fact that the low-mass portions (between 12,300 and 12,350 Da) of both experimental peaks rise almost in parallel (Figure 5d). 
Scenario c. As a third possibility we consider a scenario where protein ions exhibit different HDX properties depending on whether they are oxidized, irrespective of their charge state. For example, unoxidized $13+$ might show the same isotope exchange properties as those of unoxidized $7+$, whereas all oxidatively modified $13+$ species undergo more extensive HDX. Such a scenario is tested in Figure 5e, where only the oxygen-bearing components of $13+$ experience an additional HDX mass shift of $9 \mathrm{Da}$. The peak maxima obtained in this way are consistent with the measured data. However, the parallel rise of the experimental peaks between 12,300 and $12,350 \mathrm{Da}$ is not well reproduced by the simulation.

The simulations of Figure 5 confirm that there is a genuine difference in the experimentally observed HDX levels of high- and low-charge states after heat exposure (as in scenario b). "Trivial" mass shifts resulting from oxygen adduction (scenario $a$ ) are not large enough to account for the measured effects. We conclude that a significant subpopulation of protein in the heatexposed sample is oxidatively damaged in such a way that its members can no longer interconvert with conformers that are tightly folded. Partial unfolding of the modified proteins makes them appear in higher charge states, while at the same time giving rise to more extensive HDX. This interpretation provides a straightforward explanation for the ESI-MS and HDX behavior of cyt $c$ after heat exposure that was previously reported by Wagner and Anderegg [53].

A more subtle point is that ESI-MS also shows the presence of high-charge states for unoxidized cyt $c$ after heating, represented by the " 0 " component in Figure 3b. Charge states in this range are not observed without prior heating (Figure 1a). Thus, heat exposure seems to result in conditions that can lock protein chains in a non-native structure, even in the absence of oxidative modifications. The inadequacy of simulation scenario $c$ suggests that rapid interconversion of these species with more compact conformers does not take place. It is instructive to examine possible reasons that could cause the irreversible denaturation of unoxidized protein after heat exposure. Cyt $c$ does not possess any $-\mathrm{S}-\mathrm{S}-$ bridges, thus eliminating the possibility of disulfide shuffling [59]. A small degree of Asn or Gln deamidation $\left(-\mathrm{NH}_{2} \rightarrow-\mathrm{OH}\right.$, mass shift $=+1$ Da per affected residue) [77] cannot be excluded, although our data show that the bulk of the protein is unaffected by this process. Formation of cis isomers at the protein's four X-Pro peptide bonds may slow down the normal subsecond timescale of cyt $c$ folding [78] by several orders of magnitude [79]. However, X-Pro cis/trans isomerization is reversible on a timescale of seconds to minutes and would thus not be expected to generate proteins that are permanently locked in a non-native structure. An important point to remember is that the UV-Vis data of Figure 2a demonstrate the occurrence of partial aggregation after heating. Previous work has shown that dissociation of protein aggregates during ESI can generate highly protonated monomeric ions via asym- metric charge partitioning [20]. It is also known that interconversion between aggregated proteins and those that are free in solution is minimal [80]. In addition, conformational distortion of polypeptide chains within amorphous aggregates may promote rapid HDX. Thus, partial aggregation of the heated protein could generate a subpopulation of cyt $c$ molecules that (1) remain unoxidized, (2) appear in high-charge states, (3) do not interconvert with the native state, and (4) show a higher level of HDX than that of coexisting tightly folded conformers. Although this aggregation mechanism is a plausible scenario, other possibilities cannot be ruled out.

\section{Conclusions}

Heat exposure of cyt $c$ results in a subpopulation of irreversibly denatured protein chains-i.e., species that cannot attain their native state even when placed under "physiological" solvent conditions. The non-native molecules coexist with tightly folded species, giving rise to bimodal ESI charge-state distributions and perturbed optical spectra. This work demonstrates that the denatured conformers are heavily affected by oxidative modifications. Oxidation sites include Met65, Met80, Trp59, and the heme group. Modifications of Trp59 and Met80, in particular, are expected to be highly disruptive because in the native structure these residues are located within the tightly packed protein core [62]. Protein oxidation appears to be the main reason that prevents refolding to the native state for a considerable fraction of cyt $c$ molecules after heating. This interpretation is supported by previous reports of oxidative damage induced by different means for other proteins [71-73, 81]. With interconversion being blocked, oxidationdenatured protein chains undergo more extensive HDX than native conformers in the same solution. Unfortunately, oxygen adducts interfere with the analysis of isotope exchange data. Uncovering the presence of HDX differences requires a careful analysis of the measured peak shapes through comparisons with simulated spectra. Convolution integrals of the type used in this work (eq 2) are a useful tool for interpreting HDX data in the presence of interfering contributions. In addition to oxidation, partial aggregation appears to contribute to the formation of irreversibly denatured protein after heating. These findings represent an extension of previous work by Wagner and Anderegg [53] and Mirza et al. [55] who first reported on the interesting properties of cyt $c$ after heat exposure, without examining the physical reasons for the observed behavior.

\section{Acknowledgments}

This work was supported by the Natural Sciences and Engineering Research Council of Canada (NSERC), the Canada Foundation for Innovation (CFI), and the Canada Research Chairs Program. Marcelo O. Crespin and Shanshan Gao were involved in preliminary experiments. $\mathrm{CD}$ and fluorescence experiments were carried 
out at the UWO Biomolecular Interactions and Conformations Facility.

\section{References}

1. Kaltashov, I. A.; Eyles, S. J. Studies of Biomolecular Conformations and Conformational Dynamics by Mass Spectrometry. Mass Spectrom. Rev. 2002, 21, 37-71

2. de la Mora, F. J. Electrospray Ionization of Large Multiply Charged Species Proceeds via Dole's Charged Residue Mechanism. Anal. Chim. Acta 2000, 406, 93-104

3. Felitsyn, N.; Peschke, M.; Kebarle, P. Origin and Number of Charges Observed on Multiply-Protonated Native Proteins Produced by ESI. Int J. Mass Spectrom. Ion Process. 2002, 219, 39-62.

4. Nesatyy, V. J.; Suter, M. J.-F. On the Conformation-Dependent Neutralization Theory and Charging of Individual Proteins and Their Noncovalent Complexes in the Gas Phase. J. Mass Spectrom. 2004, 39, 93-97.

5. Iavarone, A. T.; Williams, E. R. Mechanism of Charging and Supercharging Molecules in Electrospray Ionization. J. Am. Chem. Soc. 2003, 125, 2319-2327

6. Heck, A. J. R.; Van den Heuvel, R. H. H. Investigation of Intact Protein Complexes by Mass Spectrometry. Mass Spectrom. Rev. 2004, 23, 368389.

7. Hogan, C. J.; Carroll, J. A.; Rohrs, H. W.; Biswas, P.; Gross, M. L. Charge Carrier Field Emission Determines the Number of Charges on Native State Proteins in Electrospray Ionization. J. Am. Chem. Soc. 2008, 130, 6929-6927.

8. Samalikova, M.; Grandori, R. Protein Charge-State Distributions in Electrospray-Ionization Mass Spectrometry Do Not Appear To Be Limited by the Surface Tension of the Solvent. J. Am. Chem. Soc. 2003, $125,13352-13353$

9. Nguyen, S.; Fenn, J. B. Gas-Phase Ions of Solute Species from Charged Droplets of Solutions. Proc. Natl. Acad. Sci. U. S. A. 2007, 104, 1111-1117.

10. Chowdhury, S. K.; Katta, V.; Chait, B. T. Probing Conformational Changes in Proteins by Mass Spectrometry. J. Am. Chem. Soc. 1990, 112, 9012-9013.

11. Konermann, L.; Douglas, D. J. Acid-Induced Unfolding of Cytochrome $c$ at Different Methanol Concentrations: Electrospray Ionization Mass Spectrometry Specifically Monitors Changes in the Tertiary Structure. Biochemistry 1997, 36, 12296-12302.

12. Grandori, R.; Matecko, I.; Muller, N. Uncoupled Analysis of Secondary and Tertiary Protein Structure by Circular Dichroism and Electrospray Ionization Mass Spectrometry. J. Mass Spectrom. 2002, 37, 191-196.

13. Loo, J. A.; Edmonds, C. G.; Udseh, H. R.; Smith, R. D. Effect of Reducing Disulfide-Containing Proteins on Electrospray Ionisation Mass Spectra. Anal. Chem. 1990, 62, 693-698.

14. Lin, H.; Dass, C. Conformational Changes in $\beta$-Endorphin as Studied by Electrospray Ionization Mass Spectrometry. Rapid Commun. Mass Spectrom. 2001, 15, 2341-2346.

15. Kaltashov, I. A.; Abzalimov, R. R. Do Ionic Charges in ESI MS Provide Useful Information on Macromolecular Structure? J. Am. Soc. Mass Spectrom. 2008, 19, 1239-1246.

16. Cai, X.; Dass, C. Conformational Analysis of Proteins and Peptides. Curr. Org. Chem. 2003, 7, 1841-8154

17. Katta, V.; Chait, B. T. Observation of the Heme-Globin Complex in Native Myoglobin by Electrospray-Ionisation Mass Spectrometry. J. Am. Chem. Soc. 1991, 113, 8534-8535.

18. Grandori, R. Origin of the Conformation Dependence of Protein Charge-State Distributions in Electrospray Ionization Mass Spectrometry. J. Mass Spectrom. 2003, 38, 11-15.

19. Wu, J.; Lebrilla, C. B. Intrinsic Basicity of Oligomeric Peptides that Contain Glycine, Alanine, and Valine: The Effects of the Alkyl Side Chain on Proton Transfer Reactions. J. Am. Soc. Mass Spectrom. 1995, 6, 91-101.

20. Abzalimov, R. R.; Frimpong, A. K.; Kaltashov, I. A. Gas-Phase Processes and Measurements of Macromolecular Properties in Solution: On the Possibility of False Positive and False Negative Signals of Protein Unfolding. Int. J. Mass Spectrom. 2006, 253, 207-216.

21. Schnier, P. D.; Gross, D. S.; Williams, E. R. On the Maximum Charge State and Proton Transfer Reactivity of Peptide and Protein Ions Formed by Electrospray Ionization. J. Am. Soc. Mass Spectrom. 1995, 6, 1086-1097.

22. Samalikova, M.; Grandori, R. Role of Opposite Charge in Protein Electrospray Ionization Mass Spectrometry. J. Mass Spectrom. 2003, 38, 941-947.

23. Konermann, L. A Minimalist Model for Exploring Conformational Effects on the Electrospray Charge State Distribution of Proteins. J. Phys. Chem. B 2007, 111, 6534-6543.

24. Kaltashov, I. A.; Mohimen, A. Estimates of Protein Surface Area in Solution by Electrospray Ionization Mass Spectrometry. Anal. Chem. 2005, 77, 5370-5379.

25. Grandori, R. Detecting Equilibrium Cytochrome $c$ Folding Intermediates by Electrospray Ionization Mass Spectrometry: Two Partially Folded Forms Populate the Molten Globule State. Protein Sci. 2002, 11, 453-458.

26. Mohimen, A.; Dobo, A.; Hoerner, J. K.; Kaltashov, I. A. A Chemometric Approach to Detection and Characterization of Multiple Protein Con- formers in Solution Using Electrospray Ionization Mass Spectrometry. Anal. Chem. 2003, 75, 4139-4147.

27. Yin, S.; Xie, Y.; Loo, J. A. Mass Spectrometry of Protein-Ligand Complexes: Enhanced Gas-Phase Stability of Ribonuclease-Nucleotide Complexes. J. Am. Soc. Mass Spectrom. 2008, 19, 1199-1208.

28. Benesch, J. L. P.; Ruotolo, B. T.; Simmons, D. A.; Robinson, C. V. Protein Complexes in the Gas Phase: Technology for Structural Genomics and Proteomics. Chem. Rev. 2007, 107, 3544-3567.

29. Wang, W.; Kitova, E. N.; Klassen, J. S. Influence of Solution and Gas Phase Processes on Protein-Carbohydrate Binding Affinities Determined by Nanoelectrospray Fourier Transform Ion Cyclotron Resonance Mass Spectrometry. Anal. Chem. 2003, 75, 4945-4955.

30. Feng, R.; Konishi, Y. Stepwise Refolding of Acid-Denatured Myoglobin: Evidence from Electrospray Mass Spectrometry. J. Am. Soc. Mass Spectrom. 1993, 4, 638-645.

31. Vis, H. Heinemann, U. Dobson, C. M.; Robinson, C. V. Detection of a Monomeric Intermediate Associated with Dimerization of Protein $\mathrm{Hu}$ by Mass Spectrometry. J. Am. Chem. Soc. 1998, 120, 6427-6428.

32. Wilson, D. J.; Rafferty, S. P.; Konermann, L. Kinetic Unfolding Mechanism of the Inducible Nitric Oxide Synthase Oxygenase Domain Determined by Time-Resolved Electrospray Mass Spectrometry. Biochemistry 2005, 44, 2276-2283.

33. Nabuchi, Y.; Murao, N.; Asoh, Y.; Takayama, M. Probing the Unfolding and Refolding Processes of Carbonic Anhydrase 2 Using Electrospray Ionization Mass Spectrometry Combined with $\mathrm{pH}$ Jump. Anal. Chem. 2007, 79, 8342-8349.

34. Smith, A. M.; Jahn, T. R.; Ashcroft, A. E.; Radford, S. E. Direct Observation of Oligomeric Species Formed in the Early Stages of Amyloid Formation Using Electrospray Ionization Mass Spectrometry. J. Mol. Biol. 2006, 364, 9-19.

35. Nemirovskiy, O. V.; Ramanathan, R.; Gross, M. L. Investigation of Calcium-Induced, Noncovalent Association of Calmodulin with Melittin by Electrospray Ionization Mass Spectrometry. J. Am. Soc. Mass Spectrom. 1997, 8, 809-812.

36. Invernizzi, G.; Samalikova, M.; Brocca, S.; Lotti, M.; Molinari, H. Grandori, R. Comparison of Bovine and Porcine $\beta$-Lactoglobulin: A Mass Spectrometric Analysis. J. Mass Spectrom. 2006, 41, 717-727.

37. Borysik, A. J. H.; Read, P.; Little, D. R.; Bateman, R. H.; Radford, S. E.; Ashcroft, A. E. Separation of 32 -Microglobulin Conformers by HighField Asymmetric Waveform Ion Mobility Spectrometry (FAIMS) Coupled to Electrospray Ionisation Mass Spectrometry. Rapid Commun. Mass Spectrom. 2004, 18, 2229-2234.

38. Smith, D. P.; Giles, K.; Bateman, R. H.; Radford, S. E.; Ashcroft, A. E Monitoring Copopulated Conformational States During Protein Folding Events Using Electrospray Ionization-Ion Mobility Spectrometry-Mass Spectrometry. J. Am. Soc. Mass Spectrom. 2007, 18, 2180-2190.

39. Katta, V.; Chait, B. T. Hydrogen/Deuterium Exchange Electrospray Ionisation Mass Spectrometry: A Method for Probing Protein Conformational Changes in Solution. J. Am. Chem. Soc. 1993, 115, 6317-6321.

40. Anderegg, R. J.; Wagner, D. S. Mass Spectrometric Characterization of a Protein-Ligand Interaction. J. Am. Chem. Soc. 1995, 117, 1374-1377.

41. Jaquinod, M.; Guy, P.; Halgand, F.; Caffrey, M.; Fitch, J.; Cusanovich, M.; Forest, E. Stability Study of Rhodobacter capsulatus Ferrocytochrome c2 Wild-Type and Site-Directed Mutants Using Hydrogen/Deuterium Exchange Monitored by Electrospray Ionization Mass Spectrometry. FEBS Lett. 1996, 380, 44-48.

42. Nemirovskiy, O.; Giblin, D. E.; Gross, M. L. Electrospray Ionization Mass Spectrometry and Hydrogen/Deuterium Exchange for Probing the Interaction of Calmodulin with Calcium. J. Am. Soc. Mass Spectrom. 1999, 10, 711-718

43. Krishna, M. M. G.; Hoang, L.; Lin, Y.; Englander, S. W. Hydrogen Exchange Methods to Study Protein Folding. Methods 2004, 34, 51-64.

44. Maier, C. S.; Schimerlik, M. I.; Deinzer, M. L. Thermal Denaturation of Escherichia coli Thioredoxin Studied by Hydrogen/Deuterium Exchange and Electrospray Ionization Mass Spectrometry: Monitoring a TwoState Protein Unfolding Transition. Biochemistry 1999, 38, 1136-1143.

45. Kim, M.-Y.; Maier, C. S.; Reed, D. J.; Deinzer, M. L. Conformational Changes in Chemically Modified Escherichia coli Thioredoxin Monitored by H/D Exchange and Electrospray Mass Spectrometry. Protein Sci. 2002, 11, 1320-1329.

46. Eyles, S. J.; Dresch, T.; Gierasch, L. M.; Kaltashov, I. A. Unfolding Dynamics of a $\beta$-Sheet Protein Studied by Mass Spectrometry. J. Mass Spectrom. 1999, 34, 1289-1295.

47. Simmons, D. A.; Dunn, S. D.; Konermann, L. Conformational Dynamics of Partially Denatured Myoglobin Studied by Time-Resolved Electrospray Mass Spectrometry with Online Hydrogen-Deuterium Exchange. Biochemistry 2003, 42, 5896-5905.

48. Pan, J. X.; Wilson, D. J.; Konermann, L. Pulsed Hydrogen Exchange and Electrospray Charge-State Distribution as Complementary Probes of Protein Structure in Kinetic Experiments: Implications for Ubiquitin Folding. Biochemistry 2005, 44, 8627-8633.

49. Babu, K. R.; Douglas, D. J. Methanol-Induced Conformations of Myoglobin at pH 4.0. Biochemistry 2000, 39, 14702-14710.

50. Babu, K. R.; Moradian, A.; Douglas, D. J. The Methanol-Induced Conformational Transitions of $\beta$-Lactoglobulin, Cytochrome $\mathrm{c}$, and Ubiquitin at Low pH: A Study by Electrospray Ionization Mass Spectrometry. J. Am. Soc. Mass Spectrom. 2001, 12, 317-328.

51. Wang, F.; Tang, X. Conformational Heterogeneity and Stability of Apomyoglobin Studied by Hydrogen/Deuterium Exchange and Elec- 
trospray Ionization Mass Spectrometry. Biochemistry 1996, 35, 40694078.

52. Bai, Y.; Sosnick, T. R.; Mayne, L.; Englander, S. W. Protein Folding Intermediates: Native State Hydrogen Exchange. Science 1995, 269, 192-197.

53. Wagner, D. S.; Anderegg, R. J. Conformation of Cytochrome c Studied by Deuterium Exchange-Electrospray Ionization Mass Spectrometry. Anal. Chem. 1994, 66, 706-711.

54. Anfinsen, C. B. Principles That Govern the Folding of Protein Chains. Science 1973, 181, 223-230.

55. Mirza, U. A.; Cohen, S. L.; Chait, B. T. Heat-Induced Conformational Changes in Proteins Studied by Electrospray Ionisation Mass Spectrometry. Anal. Chem. 1993, 65, 1-6.

56. Potekhin, S. A.; Kovrigin, E. L. Folding under Inequilibrium Conditions as Possible Reason for Partial Irreversibility of Heat-Denatured Proteins: Computer Simulation Study. Biophys. Chem. 1998, 73, 241-248.

57. Kreimer, D. I.; Shnyrov, V. L.; Villar, E.; Silman, I.; Weiner, L. Irreversible Thermal Denaturation of Torpedo californica Acetylcholinesterase. Protein Sci. 1995, 4, 2349-2357.

58. Miller, S.; Schuler, B.; Seckler, R. Phage P22 Tailspike Protein: Removal of Head-Binding Domain Unmasks Effects of Folding Mutations on Native-State Thermal Stability. Protein Sci. 1998, 7, 2223-2232.

59. Tani, F.; Shirai, N.; Onishi, T.; Venelle, F.; Kyoden, Y.; Doi, E. Temperature Control for Kinetic Refolding of Heat-Denatured Ovalbumin. Protein Sci. 1997, 6, 1491-1502.

60. Boys, B. L.; Konermann, L. A Temperature-Jump Stopped-Flow System of Monitoring Chemical Kinetics Triggered by Rapid Cooling. Talanta 2007, 71, 1276-1281.

61. Benjwal, S.; Verma, S.; Röhm, K.-H.; Gursky, O. Monitoring Protein Aggregation during Thermal Unfolding in Circular Dichroism Experiments. Protein Sci. 2006, 15, 635-639.

62. Mirkin, N.; Jaconcic, J.; Stojanoff, V.; Moreno, A. High Resolution X-ray Crystallographic Structure of Bovine Heart Cytochrome c and Its Application to the Design of an Electron Transfer Biosensor. Proteins Struct. Funct. Genet. 2008, 70, 83-92.

63. Babul, J.; Stellwagen, W. Participation of the Protein Ligands in the Folding of Cytochrome c. Biochemistry 1972, 7, 1195-1200.

64. Ferguson, P. L.; Pan, J.; Wilson, D. J.; Dempsey, B.; Lajoie, G.; Shilton, B.; Konermann, L. Hydrogen/Deuterium Scrambling During QuadrupoleTime-of-Flight MS/MS Analysis of a Zinc-Binding Protein Domain. Anal. Chem. 2007, 79, 153-160.

65. Bychkova, V. E.; Dujsekina, A. E.; Klenin, S. I.; Tiktopulo, E. I.; Uversky, V. N.; Ptitsyn, O. B. Molten Globule-Like State of Cytochrome c under Conditions Simulating Those Near the Membrane Surface. Biochemistry 1996, 35, 6058-6063.

66. March, R.; Todd, J. F. J. Quadrupole Ion Trap Mass Spectrometry, 2nd ed.; Wiley: New York, 2005.
67. Brahms, S.; Brahms, J. Determination of Protein Secondary Structure in Solution by Vacuum Ultraviolet Circular Dichroism. J. Mol. Biol. 1980, $138,149-178$

68. Sosnick, T. R.; Mayne, L.; Englander, S. W. Molecular Collapse: The Rate-Limiting Step in Two-State Cytochrome c Folding. Proteins Struct. Biol. Genet. 1996, 24, 413-426.

69. Xu, G.; Chance, M. R. Hydroxyl Radical-Mediated Modification of Proteins as Probes for Structural Proteomics. Chem. Rev. 2007, 107, 3514-3543

70. Tong, X.; Wren, J. C.; Konermann, L. $\gamma$-Ray-Mediated Oxidative Labeling for Detecting Protein Conformational Changes by Electrospray Mass Spectrometry. Anal. Chem. 2008, 80, 2222-2231.

71. Sharp, J. S.; Tomer, K. B. Analysis of the Oxidative Damage-Induced Conformational Changes of Apo- and Holocalmodulin by Dose-Dependent Protein Oxidative Surface Mapping. Biophys. J. 2007, 92, 1682-1692.

72. Shum, W.-K.; Maleknia, S. D.; Downard, K. M. Onset of Oxidative Damage in $\alpha$-Crystallin by Radical Probe Mass Spectrometry. Anal. Biochem. 2005, 344, 247-256.

73. Chao, C.-C.; Ma, Y.-S.; Stadtman, E. R. Modification of Protein Surface Hydrophobicity and Methionine Oxidation by Oxidative Systems. Proc. Natl. Acad. Sci. U. S. A. 1997, 94, 2969-2974.

74. Li, Y.-T.; Hsieh, Y.-L.; Henion, J. D.; Ganem, B. Studies on Heme Binding in Myoglobin, Hemoglobin, and Cytochrome c by Ion Spray Mass Spectrometry. J. Am. Soc. Mass Spectrom. 1993, 4, 631-637.

75. Sugiyama, K.; Highet, R. J.; Woods, A.; Cotter, R. J.; Osawa, Y. Hydrogen Peroxide-Mediated Alteration of the Heme Prosthetic Group of Metmyoglobin to an Iron Chlorin Product: Evidence for a Novel Oxidative Pathway. Proc. Natl. Acad. Sci. U. S. A. 1997, 94, 796-801.

76. Xiao, H.; Hoerner, J. K.; Eyles, S. J.; Dobo, A.; Voigtman, E.; Melcuk, A. I.; Kaltashov, I. A. Mapping Protein Energy Landscapes with Amide Hydrogen Exchange and Mass Spectrometry: I. A Generalized Model for a Two-State Protein and Comparison with Experiment. Protein Sci. 2005, 14, 543-557.

77. Zabrouskov, V.; Han, X.; Welker, E.; Zhai, H.; Lin, C.; van Wijk, K. J.; Scheraga, H. A.; McLafferty, F. W. Stepwise Deamidation of Ribonuclease $\mathrm{A}$ at Five Sites Determined by Top Down Mass Spectrometry. Biochemistry 2006, 45, 987-992.

78. Englander, S. W.; Sosnick, T. R.; Mayne, L. C.; Shtilterman, M.; Qi, P. X.; Bai, Y. Fast and Slow Folding in Cytochrome c. Acc. Chem. Res. 1998, 31, 737-744.

79. Houry, W. A.; Scheraga, H. A. Nature of the Unfolded State of Ribonuclease A: Effect of cis-trans X-Pro Peptide Bond Isomerisation. Biochemistry 1996, 35, 11719-11733.

80. Dobson, C. M. Protein Folding and Misfolding. Nature 2003, 426, $884-890$.

81. Tong, X.; Wren, J. C.; Konermann, L. Effects of Protein Concentration on the Extent of $\gamma$-Ray-Mediated Oxidative Labeling Studied by Electrospray Mass Spectrometry. Anal. Chem. 2007, 79, 6376-6382. 